\title{
Glucose sensing by means of silicon photonics
}

\author{
Ronny Bockstaele, Eva Ryckeboer, Nannicha Hattasan, Yannick De Koninck, Muhammed Muneeb, \\ Steven Verstuyft, Danae Delbeke, Wim Bogaerts, Gunther Roelkens, Roel Baets \\ Photonics Research Group, Ghent University - imec, Belgium, \\ Center for Nano- and Biophotonics, Ghent University, Belgium
}

\begin{abstract}
Diabetes is a fast growing metabolic disease, where monitoring the blood glucose values in combination with insulin injection is currently the only therapy to keep the glucose concentration in diabetic patients under control, minimizing the long-term effects of elevated glucose concentrations and improving quality of life of the diabetic patients. Implantable sensors allow continuous glucose monitoring, offering the most reliable data to control the glucose. Infrared spectroscopy is proposed as a non-chemical measurement method to determine the small glucose concentrations in blood serum, with promise for a long implantation time. In this work, a spectrometer platform based on silicon photonics is presented, allowing the realization of very small glucose sensors suitable for building implantable sensors. A proof-ofconcept of a spectrometer with integrated evanescent sample interface is presented, and the route towards a fully implantable spectrometer is discussed.
\end{abstract}

Keywords: Glucose, Silicon Photonics, infrared spectrometer

\section{INTRODUCTION}

Diabetes is a fast growing metabolic disease, where patients have an elevated glucose concentration. The number of diabetic patients worldwide is estimated at 347 million [1]. In healthy persons, insulin is released by the pancreas and controls the uptake of glucose by the cells. If the production of insulin is reduced, or if the cells become resistant to insulin, the uptake of glucose is disturbed, leading to multiple adverse effects for the patient: on short term, the glucose concentration is strongly fluctuating, resulting in very high or low glucose values. Hypoglycemia poses an acute risk. If the average glucose concentration is large, this can lead to many problems, such as nerve damage, kidney or eye problems, heart disease and stroke.

Measuring the blood glucose values in combination with extra injection of insulin at moments of high glucose concentration is required to keep the blood glucose concentration in normal limits. The reference method, however, is a hexokinase based method starting from the blood serum (after filtering the red blood cells). This offers the highest accuracy, but required specialized equipment. A more practical method is the finger-prick method, where a small blood droplet is taken from the patient, put on a strip including dry chemistry for a glucoxidase enzymatic reaction. This reaction converts the amount of glucose in a small current.

Implantable sensors offer more user comfort and allows for continuous glucose monitoring: the glucose is measured automatically, without need for user intervention such as finger pricking. Implantable sensors based on the enzymatic reaction are commercially available and FDA approved, from different suppliers (Dexcom, MedTronics, Menarini). These sensor consist of a small probe that contains the chemistry for the enzymatic reaction, inserted under the skin in the subcutaneous region, and connected to a readout unit. The sensors are thus transdermal, partially under the skin, partially on top of the skin. The implantation period is limited to 3-5 days (depending on the product). Two effects limit the maximal implantation time: the stability of the chemical reagents taking part in the enzymatic reaction, and the foreign body reaction to by-products of the chemistry resulting in an encapsulation of the probe.

Optical absorption spectroscopy is proposed as a method for glucose sensing as glucose has its own specific spectral features both in the first overtone band (1500-1800nm) and the combination band (2100-2400nm). However, the glucose absorption spectrum is overlapping with the spectrum of water and other interferants in the interstitial fluid. Moreover, the glucose concentration is rather small in the serum (typically glucose concentration in healthy persons is 80 $200 \mathrm{mg} / \mathrm{dL}$, corresponding to $0.008-0.02$ weight $\%$ ), implying the changes in the absorption spectrum due to glucose 
concentration variations is also small $(<0.1 \%)$. Therefore a multivariate model is used to estimate the glucose concentration from the measured absorption spectrum of the interstitial fluid.

This paper described a Silicon Photonics spectrometer system for glucose sensing, including thin-film photodiodes bonded on the photonic chip and an evanescent sample interface. Both the design, fabrication and characterization are presented.

\section{SILICON PHOTONIC SPECTROMETERS}

Silicon photonics allows for cost-effective large-volume fabrication of photonic chips [ref]. The light is guided in a thin silicon waveguide (with high refractive index) lying on a thick Silicon Oxide layer (low refractive index). The technology is used in multiple commercial applications such as telecommunication systems and biosensors [ref]. These application all use light around $1550 \mathrm{~nm}$. Glucose sensing requires measuring the absorption spectrum around $1590 \mathrm{~nm}$ and $2320 \mathrm{~nm}$. In a first step, spectrometer structures in silicon photonics sensing these wavelength bands are designed, fabricated and tested.

\subsection{Design of the dispersive element}

The wavelength demultiplexer is a planar concave grating (PCG), also known as echelle grating. This component has a small footprint for the required spectral resolution for glucose sensing $(\sim 5 \mathrm{~nm})$. The operating principle can be explained as follows (see figure 1): light from an input aperture is incident on a curved diffraction grating that will redirect and refocus the light depending on its wavelength to a certain output aperture. The light propagates to and from the grating in the free propagation region (FPR) which is a single-mode $220 \mathrm{~nm}$ high slab waveguide. The design is based on the Rowland geometry [ref1]. The grating facets are located on a circle with radius $2 \mathrm{R}$ while the in- an output apertures are positioned along the Rowland circle with radius R. An example design is shown in fig 1. The Rowland circle is clearly indicated. The apertures are shallowly $(70 \mathrm{~nm})$ etched waveguides to reduce reflections at the waveguide to slab transition. The grating facets are deeply $(220 \mathrm{~nm})$ etched first or second order distributed Bragg gratings (DBR) to enhance the reflectivity. Light is coupled in and out of the spectrometer using grating couplers. In the high index contrast silicon-on-insulator platform, small fabrication imperfections easily degrade the performance, therefore nano-scale processing accuracy is required.



Figure 1. example design of a 8 channel PCG with $3.2 \mathrm{~nm}$ resolution and central wavelength of $1550 \mathrm{~nm}$.

For a PCG, residual slab thickness variations are the main cause of crosstalk due to accumulated phase errors. More detailed information on the design and typical performance can be found in [ref2, ref3]. For glucose detection, these demultiplexers need to be designed and fabricated for both the first overtone band (1550-1850 nm) and combination band (2000-2350 nm) where glucose has absorption features. We have targeted two glucose absorption peaks with two individual PCG designs that are listed in table 1. The main design parameters and the measured performance in terms of 
cross-talk and insertion loss are tabulated. The exact cross-talk level of the combination band PCG was masked by the high noise floor of our set-up [ref3]. Next to the free spectral range of the spectrometer, the operation bandwidth of the PCG is limited by the wavelength range that can be coupled by the grating couplers. In the first overtone band, our current grating coupler design, based on straight gratings with $70 \mathrm{~nm}$ shallowly etched trenches, has a limited $3 \mathrm{~dB}$ bandwidth of 60-80 $\mathrm{nm}$ [ref4]. In the combination band, however, a straight grating with $150 \mathrm{~nm}$ thick grating lines, coupled to a $150 \mathrm{~nm}$ thick silicon waveguide offers a broader $3 \mathrm{~dB}$ wavelength range of $150 \mathrm{~nm}$ [ref3]. A linear taper is then used to convert the mode from the $150 \mathrm{~nm}$ thick waveguide to a standard $220 \mathrm{~nm}$ high silicon wire. Nonetheless, for glucose sensing, a larger spectral area needs to be covered. This means that a set of PCGs, rather than one single PCG, co-integrated on a single chip are necessary for the glucose sensing application.

Table 1. Characteristics of the silicon photonics spectrometer structures.

\begin{tabular}{|l|l|l|}
\hline \multicolumn{1}{|c|}{ Item } & \multicolumn{1}{c|}{ Design 1 } & \multicolumn{1}{c|}{ Design 2 } \\
\hline Center wavelength [nm] & $1590 \mathrm{~nm}$ & $2320 \mathrm{~nm}$ \\
\hline Resolution [nm] & 3 & $0-10$ \\
\hline Number of channels & 30 & 0.8 \\
\hline Wire width [um] & 0.45 & 3 \\
\hline Aperture width [um] & 2.5 & 60 \\
\hline Free spectral range [nm] & 107 & 540 (first order) \\
\hline DBR period [nm] & 340 (first order) & 1.04 \\
\hline Footprint [mm2] & 0.8 & $5 \mathrm{~dB}$ \\
\hline Insertion loss & $4 \mathrm{~dB}$ & $<-16 \mathrm{~dB}$ \\
\hline Cross talk & $-16.5 \mathrm{~dB}$ & \\
\hline
\end{tabular}

\subsection{Integration of photodiodes}

The readout of the spectrometer requires a light-to-current conversion. Photodiodes are integrated on the silicon photonics chip to enable this. The process involved die bonding, substrate removal, and lithographical definition of the mesa, $\mathrm{N}$ and P-contacts. Thin-film bonded photodiodes offer important advantages over standard pick-and-place mounted photodiodes: the size of the photodiodes can be very small resulting in a small capacitance and dark current for optimized devices.

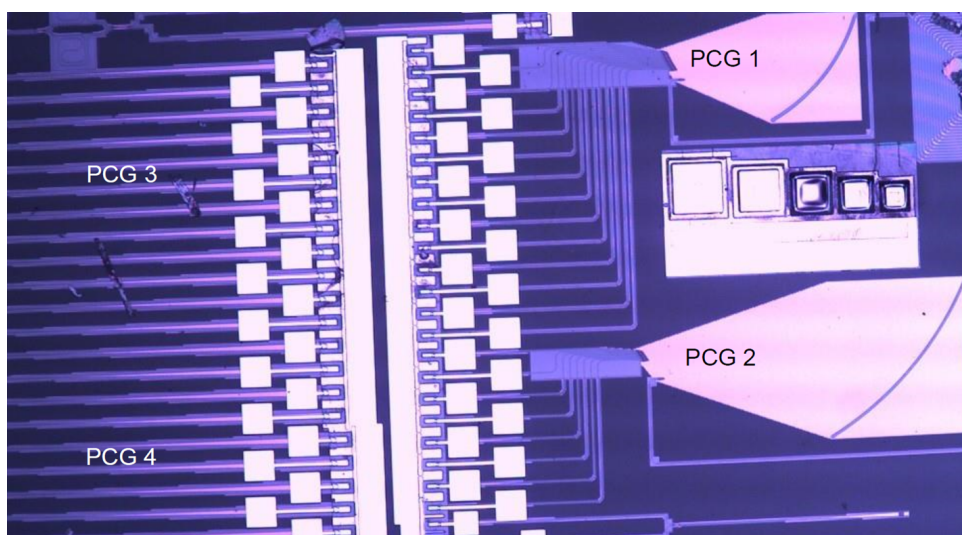


Figure 2: close-up of the silicon photonics spectrometer with integrated photodiodes. The test chip contains 4 spectrometer structures: 2 PCGs are shown, together with part of the photodiodes. The integrated photodiodes are much smaller the PCG.

The integrated photodiode arrays consist of $\mathrm{p}$-i-n diodes with $\mathrm{Ga}_{0.79} \mathrm{In}_{0.21} \mathrm{As}_{0.19} \mathrm{Sb}_{0.81}$ as an intrinsic absorbing layer. The composition of the intrinsic layer is selected to have a cut-off wavelength at $2.5 \mu \mathrm{m}$ wavelength. Therefore, these photodetectors can accommodate both first overtone band as well as the combination band wavelength range. Figure 3 presents the V-I characteristic of the photodetector at different input power levels at room temperature. An intrinsic responsivity of $0.3 \mathrm{~A} / \mathrm{W}$ is obtained. The details of epitaxy, coupling design and fabrication of these integrated photodetectors are discussed in [1][2].

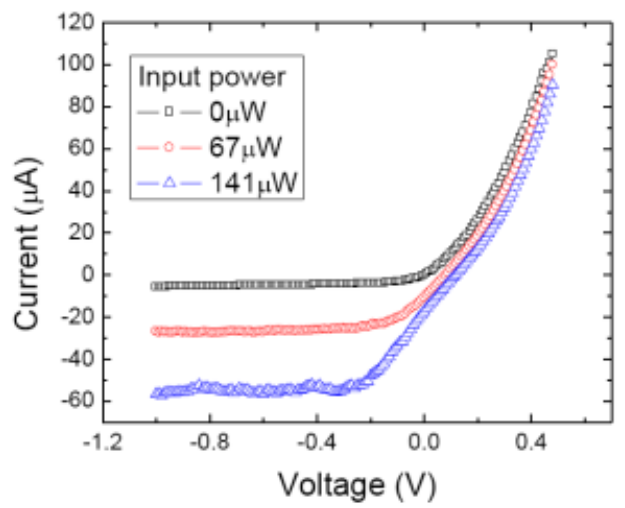

Figure 3. Photocurrent characteristics of the thin-film photodiodes bonded on the silicon photonics chips.

\subsection{Characterization of the spectrometers}

Planar concave grating devices with integrated photodiodes have been fabricated and characterized: the light of a tunable laser is coupled via a grating coupler to the spectrometer, and the photocurrent response of each photodiode is measured as function of the input wavelength. Figure 4 shows the response of all photodiodes of the spectrometer as function of the laser wavelength (superimposed on one graph). The response of the photodiodes peaks when the wavelength of the laser matches the spectral grid.
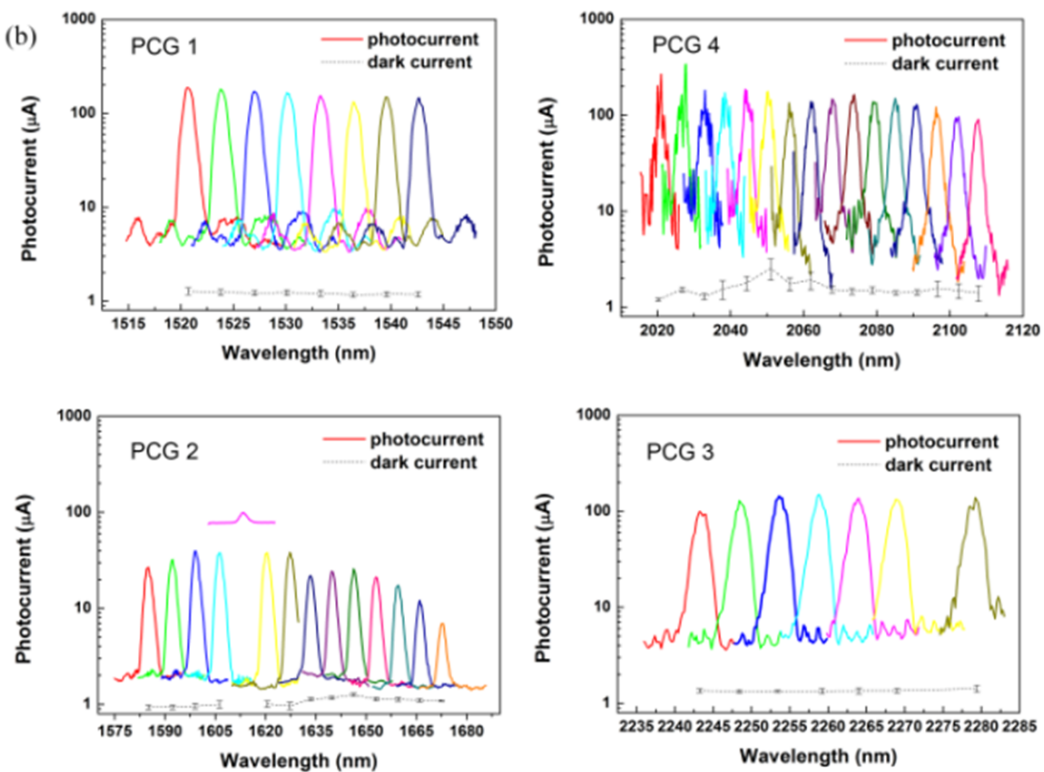

Figure 4: The optical spectral characteristics of different spectrometer structures implemented in silicon photonics. 
Spectrometers with integrated photodiodes, both for first overtone band and combination band are characterized.

\section{EVANESCENT SENSING}

An absorption spectrometer measures the spectrum of the light after propagation through the sample. In a traditional spectrometer, the sample is inside a cuvette, the light propagates through the cuvette before detection by a spectrometer. This setup allows for easy sample interfacing, but as the light spreads out during propagation through the cuvette, a large diameter beam arrives at the spectrometer input. Moreover, the light can be scattered by inhomogeneities in the sample, resulting in a variable coupling efficiency into the spectrometer. This effect is more pronounced in Silicon Photonics spectrometers, where the input light must be coupling into a single-mode waveguide.

A far more elegant approach is the evanescent absorption spectrometer, where the light remains guided in the singlemode waveguide. Part of the light is outside the waveguide (the evanescent part) and thus absorbed by the surrounding medium. In this approach a compact system for measuring the absorption spectrum can be realized.



Figure 5: the optical mode profile in the silicon waveguide. Part of the light is outside the waveguide and senses the absorption by the sample on top of the waveguide.

Evanescent sensing has important advantages compared to the traditional free-space spectrometer system: it is a very compact system, the alignment from source to sample to spectrometer is defined by the photonics circuit and thus not sensitive to mechanical vibrations or changes in the alignment. The waveguide approach can be easily extended to multiple paths, even with a different path lengths, by introduction waveguide splitters and combiners in the photonic chip. The sensed volume is extremely small: the evanescent penetration depth is typically $50-100 \mathrm{~nm}$, depending on the wavelength and the dimensions and material properties of the waveguide.

The feasibility of using an evanescent interface for measuring the very small changes in the transmission spectrum due to glucose concentration variation is reported in []. A tunable light source is coupled to the silicon photonics waveguide, and read-out by an external optical power meter. The evanescent waveguide sensor is based on a wire waveguide, the TE mode is guided and thus the mode that feels the absorption by the sample on top of the waveguide. Glucose concentrations down to $1 \mathrm{mM}$ have been measured.

\section{ABSORPTION SPECTROMETER MEASUREMENTS}

Structures with waveguides for evanescent absorption spectrometry, coupled to on-chip spectrometer devices for measuring the absorption spectrum were fabricated. Figure 6 shows the design of the fabricated devices. 


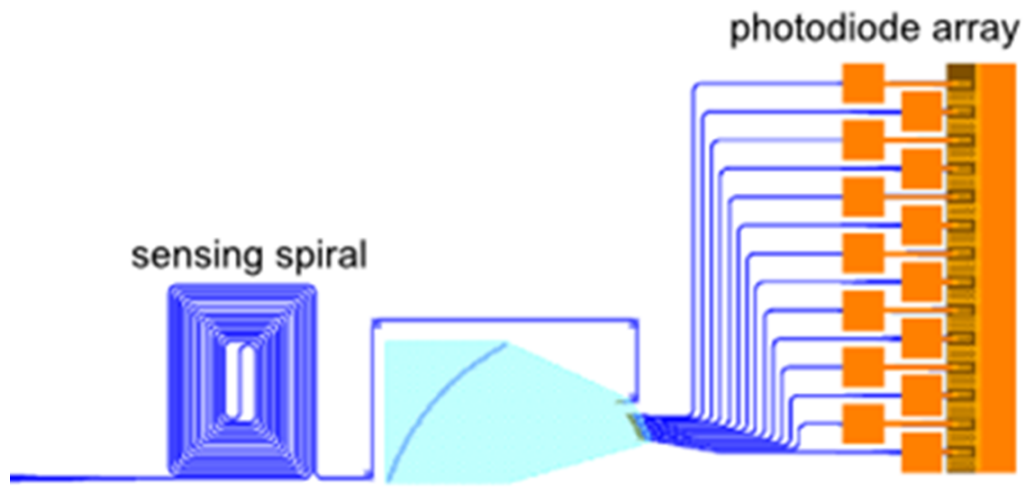

Figure 6. Combining the sensing spiral, the planar concave grating and the photodiode array in an integrated spectrometer device.

To measure the absorption spectrum, an SLED light source (Covega) with polarization maintaining output fiber is connected to a 3-wheel polarization controller and further to the SOI chip using polarization maintaining fiber. A probe card connects to the anode and cathode electrical contacts of the photodiodes in the array. A custom-built multi-channel trans-impedance amplifier board converts the photocurrent to a voltage. A NI AD box converts the voltage into a digital signal. A Python script controls the readout.
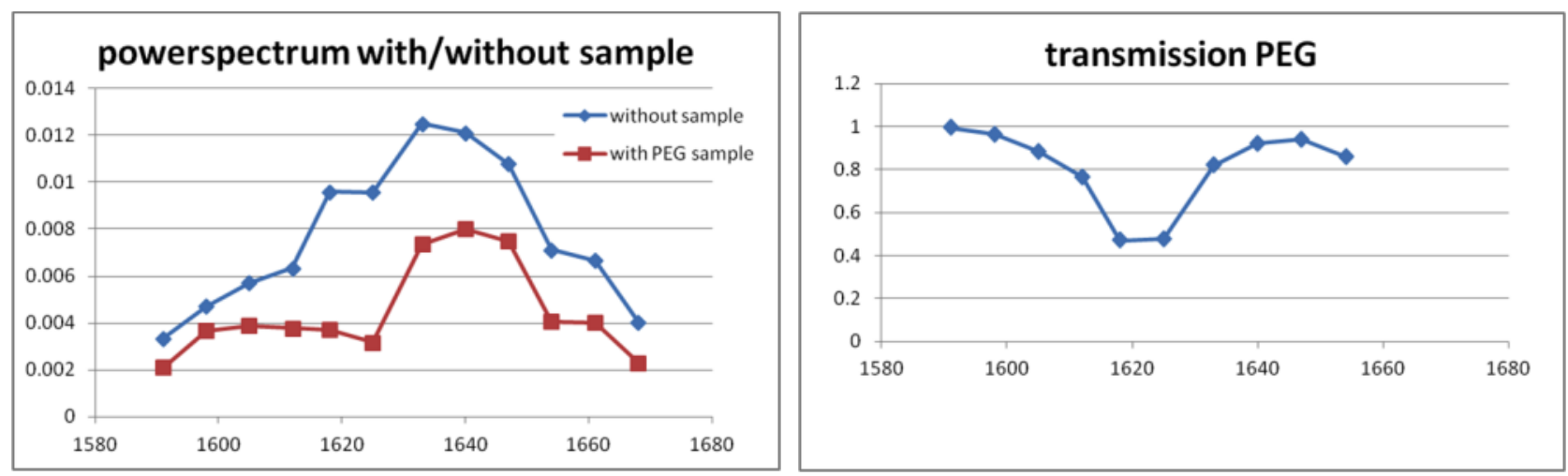

Figure 7: the spectrum of the SLED source without (blue) and with (red) sample on the sensing spiral (left), and the calculated transmission spectrum (right).

The response of the silicon photonics spectrometer is measured without and with liquid sample on the sensing spiral. The ratio of the two spectra is the absorption spectrum. Poly-ethylene Glycol (PEG) is used as test sample, as this substance has a specific absorption peak in the first overtone band at $1620 \mathrm{~nm}$, in the center of the spectrometer measurement range. The absorption spectrum measured is in agreement with the theoretical absorption spectrum of PEG. The spectrum is shown in figure 7.

\section{INTEGRATION OF LIGHT SOURCES IN THE SPECTROMETER}

Silicon photonics spectrometers including photodiodes and sample interfaces have been demonstrated. As noticed in the introduction, the changes in absorption spectrum due to glucose is clinical applications is very small. The principle of using evanescent light absorption to measure glucose concentration variations for measuring glucose is reported elsewhere [ref], showing the feasibility of this type of setup for measuring the ultra-small variations in absorption spectrum corresponding to the changes of blood glucose in clinical applications. The last missing link is a light source integrated integrated on the chip. A broadband light source is required to illuminate the sample. In this work a laser array is selected as light source, and two implementations are investigated. The integration of the laser on the silicon photonics 
is similar to the integration of photodiodes: the III/V epitaxy is adhesively bonded on the silicon photonics chip, the substrate is removed and the laser structures are defined lithographically in the remaining III/V layer on the silicon photonics chip.

\section{Electrically pumped hybrid InGaAsSb-Si laser for the combination band}

The laser is designed to have the optical mode divided between the $\mathrm{Si}$ waveguide and a gain region $\left(\mathrm{In}_{0.67} \mathrm{Ga}_{0.33} \mathrm{As}_{0.08} \mathrm{Sb}_{0.92}\right.$ quantum wells). Figure 8 illustrates the device schematically (not-to-scale). The 1D optical mode profile is presented in Figure NH2b. With such an optical mode design, it allows an efficient light coupling from laser to $\mathrm{Si}$ waveguide. Moreover, it is possible to form wavelength selective components such as gratings on the Si to provide feedback. Therefore, single wavelength lasers such as DFB lasers can be achieved with this design. Figure NH2c presents the preliminary L-I measurement result of the hybrid InGaAsSb-Si Fabry-Perot laser. The laser operates up to $10^{\circ} \mathrm{C}$ in pulsed regime. The optical spectrum is shown in the inset. The wavelength emission is at $\sim 2.4 \mu \mathrm{m}$
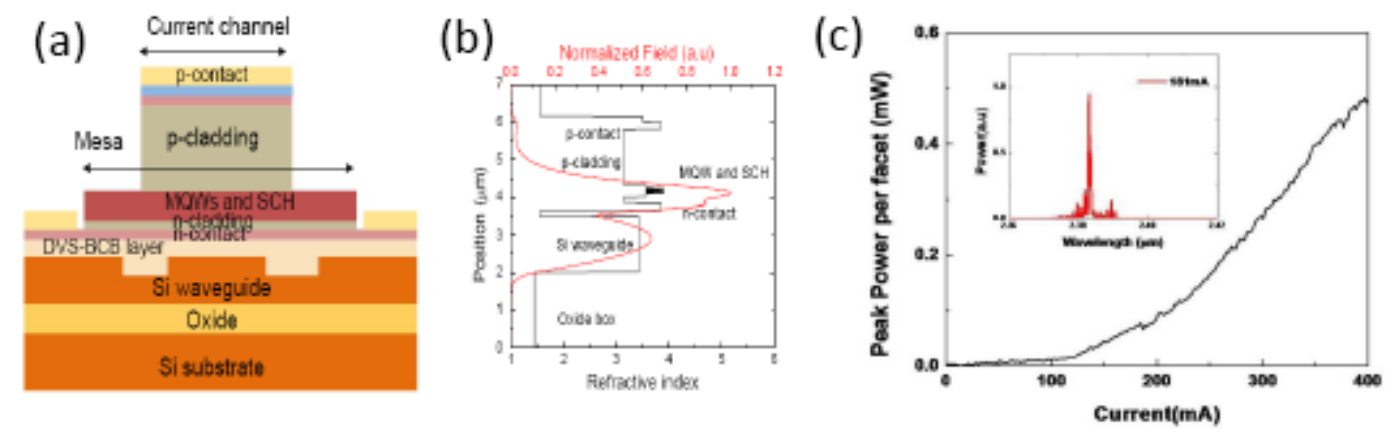

Figure 8: a) Not-to-scale device schematic b) $1 \mathrm{D}$ optical mode profile $(\lambda=2.42 \mu \mathrm{m}$, TE-polarization) c) L-I characteristic of the realized laser measured in pulsed regime at $10^{\circ} \mathrm{C}$. The inset presents the optical spectrum.

\section{Electrically pumped hybrid InGaAsSb-Si laser for combination band}

A novel microlaser type based on heterogeneous integration of III-V materials onto the SOI circuit using adhesive bonding was developed. The novel laser relies on resonant silicon grating cavities buried under the III-V laser mesa that act as narrow-band reflectors [REF theoretical paper]. This approach allows for small, single mode laser devices that require low threshold power. Recently we demonstrated an optically pumped prototype of this device [REF exp. paper]. This laser is $55 \mathrm{um}$ long, requires $\pm 2 \mathrm{~mW}$ threshold power and had a side-mode suppression ratio of $39 \mathrm{~dB}$. An electrically pumped version of this laser is being developed.

\section{CONCLUSIONS}

The development of a Silicon Photonics spectrometer platform for implantable glucose sensing applications is presented. The platform includes multi-wavelength light sources based on laser arrays, evanescent sample interface, optical spectrometers and an array of thin-film bonded photodiodes. Spectrometer structures for spectral analysis of light centered round $1590 \mathrm{~nm}$ and $2320 \mathrm{~nm}$ is reported, covering the two spectral bands where the glucose absorption can be distinguished from the absorption of the water and the other constituents in the blood serum. This work demonstrates the feasibility of ultra-small spectrometer systems, suitable for integration in a biocompatible package to realize a long-term implantable glucose sensor. This type of sensors will help diabetic patients in controlling their blood glucose values, improving their quality-of-life. 


\section{REFERENCES}

[1] Booth, N. and Smith, A. S., [Infrared Detectors], Goodwin House Publishers, New York \& Boston, 241-248 (1997).

[2] Davis, A. R., Bush, C., Harvey, J. C. and Foley, M. F., "Fresnel lenses in rear projection displays," SID Int. Symp. Digest Tech. Papers 32(1), 934-937 (2001).

[3] [ref1]= R. Marz. Integrated optics, Design and Modeling. (Artech House Inc., 1994).

[4] [ref2] = J. Brouckaert, W. Bogaerts,P. Dumon, D.Van Thourhout, and R. Baets, "Planar concave grating demultiplexer fabricated on a nanophotonic silicon-on-insulator platform," J. Lightwave Technol. 25(5), 12691275 (2007).

[5] [ref3]= E.M.P. Ryckeboer, A. Gassenq, M. Muneeb, N. Hattasan, S. Pathak, L. Cerutti, J-B Rodriguez, E. Tournie, W. Bogaerts, R. Baets, G. Roelkens, Silicon-on-insulator spectrometers with integrated GaInAsSb photodiodes for wide-band spectroscopy from 1510 to $2300 \mathrm{~nm}$, Optics Express, (2013)

[6] [ref4]= G. Roelkens, D. Vermeulen, D. Van Thourhout, R. Baets, S. Brision, P. Lyan, P. Gautier, J.-M. Fedeli, High efficiency diffractive grating couplers for interfacing a single mode optical fiber with a nanophotonic silicon-on-insulator waveguide circuit, Applied Physics Letters, 92(13), p.131101 (2008)Van Derlofske, J. F., "Computer modeling of LED light pipe systems for uniform display illumination," Proc. SPIE 4445, 119-129 (2001).

[7] Myhrvold, N., "Confessions of a cybershaman," Slate, 12 June 1997, <http://www.slate.com/CriticalMass/9706-12/CriticalMass.asp > (19 October 1997). www.optics4yurresearch.com/7752.html

[8] Jones, C. J., Director, Miscellaneous Optics Corporation, interview, Sept. 232011

[9] FamilyName, GivenName Initial., "Title," Source, pg\# (year). 